\title{
sciendo
}

\author{
BULGARIAN ACADEMY OF SCIENCES
}

CYBERNETICS AND INFORMATION TECHNOLOGIES • Volume 20, No 6

Special Issue on New Developments in Scalable Computing

Sofia $\bullet 2020$

Print ISSN: 1311-9702; Online ISSN: 1314-4081

DOI: $10.2478 /$ cait-2020-0065

\section{Numerical Solution of Integro-Differential Equations Modelling the Dynamic Behavior of a Nano-Cracked Viscoelastic Half-Plane}

\author{
Tsviatko V. Rangelov ${ }^{1}$, Petia S. Dineva ${ }^{2}$, George D. Manolis ${ }^{3}$ \\ ${ }^{1}$ Institute of Mathematics and Informatics, Bulgarian Academy of Sciences, 1113 Sofia, Bulgaria \\ ${ }^{2}$ Institute of Mechanics, Bulgarian Academy of Sciences, 1113 Sofia, Bulgaria \\ ${ }^{3}$ Department of Civil Engineering, Aristotle University, Thessaloniki GR-54124, Greece \\ E-mails: rangelov@math.bas.bg petia@imbm.bas.bg gdm@civil.auth.gr
}

\begin{abstract}
The scattering of time-harmonic waves by a finite, blunt nano-crack in a graded, viscoelastic bulk material with a free surface is considered in this work. Nonclassical boundary conditions and a localized constitutive equation at the interface between crack and matrix, following the Gurtin-Murdoch surface elasticity theory are introduced. An efficient numerical technique is developed using integrodifferential equations along the nano-crack line that is based on an analytically derived Green's function for the quadratically inhomogeneous half-plane. The dependence of the diffracted and scattered waves and of the local stress concentration fields on key problem parameters such as viscosity, inhomogeneity, surface elasticity, and interaction between the nano-crack and the free surface are all examined through an extensive parametric study.
\end{abstract}

Keywords: Viscoelasticity, graded half-plane, nano-crack, integro-differential equations, stress concentration, wave scattering, wave diffraction.

\section{Introduction}

The aim of the study is to solve the problem of dynamic fracture for a nano-cracked, viscoelastic and inhomogeneous half-plane subjected to time-harmonic waves by the Boundary Integral Equation Method (BIEM). This numerical method is based on solution of an integro-differential equation along the nano-crack interface, with kernel functions which are derived analytically in the form of a Green's function and its derivatives for the inhomogeneous half-plane, see Rangelov and Manolis [13]. The study is a continuation of the authors' previous results, see R a n g e lo v, Dine va and Manolis [12], where the dynamic nano-crack problem is solved in a viscoelastic anisotropic plane, and in R a n e lo v, Din e va and M a n ol is [11], where the dynamic nano-crack problem is solved in an elastic isotropic graded halfplane. 
The novelty of the current work lies in:

(a) The use of conventional mechanical models with the appropriate modifications to account for surface effects produces software that is efficient, both in terms of time plus memory requirements. This is in contrast to more advanced continuum mechanics models [1] that consider the atomic level, which in turn yields software with very high computational requirements.

(b) The solution of integro-differential equations for a more sophisticated scalable and accurate mechanical model that takes into account material viscosity using the Zener model, plus the quadratic variation of the material parameters with depth.

We introduce the use of a dynamic Green's function for the graded half-plane, as well as the free-field wave solution in an inhomogeneous half-plane. Finally, numerical examples are presented for the Stress Concentration Field (SCF) near the nano-crack tip and the scattered wave field along the free-surface of the graded halfplane, so as to demonstrate the influence of all these mechanical model parameters on the problem at hand.

\section{Statement of the problem}

Consider an isotropic, viscoelastic, quadratically inhomogeneous in depth half-plane defined in the Cartesian coordinate system $O x_{1} x_{2}$ as the domain $\Omega=\left\{x_{2}<0\right\}$ $d / 2 a=1.5$, with free-surface boundary $\Gamma_{\mathrm{ff}}=\left\{x_{2}=0\right\}$. The domain $\Omega$ contains an embedded nano-crack at fixed depth $d$ under normally incident, free field timeharmonic

P-waves with frequency $\omega$ propagating in the half-plane $x_{2}<0$. In-plane wave motion with respect to the plane $x_{3}=0$ is assumed, see Fig. 1. The model for the nano-crack $S$ is a blunt crack with a crack root in the shape of a semi-ellipse with semi-major axis $c_{0}$ and semi-minor axis $c, c \leq c_{0}$ (Fig.1). The perimeter of the crack is $|S|=2\left(2 a-2 c_{0}\right)+4 c_{0} E(g), c_{0}=0.0375 a$, with size parameter $a$ falling in the interval $10^{-7}-10^{-10} \mathrm{~m}$, where $g=\sqrt{1-\left(c / c_{0}\right)^{2}}$ and $E(g)=\int_{0}^{\pi / 2} \sqrt{1-g^{2} \sin ^{2} \varphi} d \varphi$ is the complete elliptic integral of second kind, see [4]. If $c=c_{0}$, then the blunt nano-crack has a crack root of semi-circular shape with radius $c_{0}$, while if $c=0$, the blunt nanocrack degenerates to a line crack with a length $2 a$. The blunt nano-crack's line $S$ can be expressed in the following way, see [11]: $S=S^{+} \cup S^{-} \cup S^{1} \cup S^{\mathrm{r}}$, where the flat part of $S$ is $S^{+} \cup S^{-}$, and the left and right crack roots are represented by semielliptical shapes $S^{1} \cup S^{\mathrm{r}}$. 


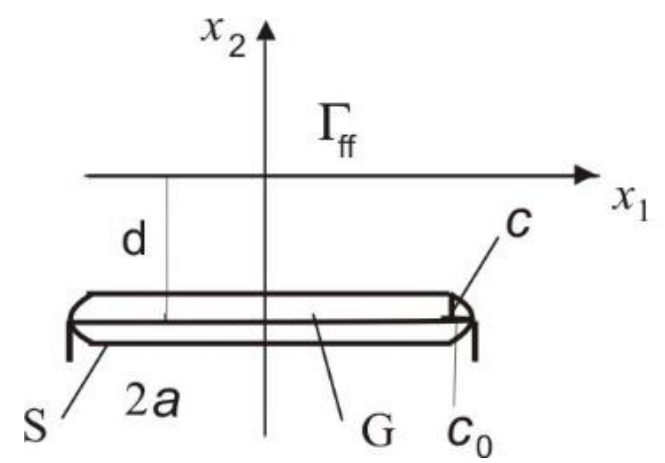

Fig. 1. Graded half-plane with a blunt nano-crack $S$ under a normally incident time-harmonic P-wave

The bulk material properties are assumed to be functions of one coordinate (say depth for convenience), i.e., $\mu(x)=\lambda(x)=\mu_{0} h(x)$, and $\rho(x)=\rho_{0} h(x)$, where function $h(x)=\left(a_{2} x_{2}+1\right)^{2}, a_{2} \leq 0$, is a quadratic function. This material profile indicates a quadratic-type variation with respect to the depth coordinate $x_{2} \leq 0$. Furthermore, Poisson's ratio is fixed at $v=0.25$ and $\mu_{0}>0, \rho_{0}>0$ are reference values for the shear modulus and the mass density, respectively, which define the corresponding homogeneous half-plane when $a_{2}=0$. Under plane-strain conditions, the only non-zero field quantities are the displacement components $u_{i}(x, \omega), i=1,2$, the stress components $\sigma_{i j}(x, \omega)$, the strain components $\varepsilon_{i j}(x, \omega)$ and the tractions $t_{i}(x, \omega)=\sigma_{i j}(x, \omega) n_{j}$. Here, $n_{j}, j=1,2$, are the components of the outward pointing, unit normal vector to either the nano-crack boundary $S$ or to the free surface $\Gamma_{\mathrm{ff}}$, and all quantities defined depend on coordinates $x=\left(x_{1}, x_{2}\right)$ and on the frequency $\omega$ of the incident wave. The Boundary-Value Problem (BVP) under consideration represents elastic wave scattering and diffraction by a sub-surface finite crack located at depth $d$ beneath the free surface $\Gamma_{\mathrm{ff}}$ of the graded half-plane. In what follows, we define the BVP in the frequency domain in terms of the governing equations of motion and the boundary conditions.

\subsection{Constitutive equations}

The constitutive law used here follows the Zener viscoelastic model with fractional time derivatives of order $\alpha$ in the frequency domain (see $S \mathrm{ch}$ a n z [14], M a i n a r d i [7]):

$$
\left(1+p(i \omega)^{\alpha}\right) \sigma_{i j}(x, \omega)=C_{i j k l}\left(1+q(i \omega)^{\alpha}\right) \varepsilon_{k l}(x, \omega),
$$

where: $p \geq 0, q \geq 0, \alpha \in[0,1]$ are the model coefficients, and for an isotropic material, $C_{i j k l}=\lambda(x) \delta_{i j} \delta_{k l}+\mu(x)\left(\delta_{i k} \delta_{j l}+\delta_{i l} \delta_{j k}\right)$ are the stiffness coefficients and $\delta_{i j}$ is the Kroneker symbol. If $\alpha=0$ or if $p=q$, then the Zener model (1) degenerates to Hooke's law for anisotropic materials. In this case $\alpha=1, p \neq q$, we have the classical Zener model presenting the rheological model for the standard linear solid. 
The derivative order denoted by $\alpha$ has no physical meaning, e.g., it simply acts as an interpolant between two ascending order integer derivatives. For instance, parameter $\alpha$ in a single degree-of freedom representation of a nonlinear base isolation system interpolates between the zero and the first order time derivatives that appear in the equation of motion, see $\mathrm{Makrou}$ and $\mathrm{Manolis}$ [8]. A best-fit (or optimal) value for $\alpha$ can only be estimated by recourse to experimentally obtained data.

Next, under the assumption of small deformations, the strain-displacement relation for in-plane wave motion is

$$
\varepsilon_{k l}(x, \omega)=0.5\left(u_{k, l}(x, \omega)+u_{l, k}(x, \omega)\right),
$$

where the comma subscripts denote partial differentiation with respect to the spatial coordinates and the summation convention is implied over repeated indices.

\subsection{Equation of motion and incident wave field}

The equations of motion for time-harmonic waves in the absence of body forces are

$$
\sigma_{i j, j}(x, \omega)+\rho(x) \tilde{\omega}^{2} u_{i}(x, \omega)=0,
$$

In the above, $\tilde{\omega}^{2}=\frac{1+p(i \omega)^{\alpha}}{1+q(i \omega)^{\alpha}} \omega^{2}$ is the square of the damped frequency, see Dine va and Rangelov [2]. The total displacement $u_{i}(x, \omega)$ and stress $\sigma_{i j}(x, \omega)$ wave fields that develop in a graded half-plane containing an embedded nano-crack are as follows:

$$
u_{i}(x, \omega)=u_{i}^{\mathrm{ff}}(x, \omega)+u_{i}^{\mathrm{sc}}(x, \omega), \sigma_{i j}(x, \omega)=\sigma_{i j}^{\mathrm{ff}}(x, \omega)+\sigma_{i j}^{\mathrm{sc}}(x, \omega),
$$

where $u_{i}^{\mathrm{ff}}(x, \omega)$ and $\sigma_{i j}^{\mathrm{ff}}(x, \omega)$, respectively, are the free-field displacements and stresses satisfying the governing Equation (3), the Sommerfeld radiation condition at infinity and the zero traction conditions along the free-surface $\Gamma_{\mathrm{ff}}$ of the graded halfplane in the absence of a crack ( $\mathrm{M}$ a noli s et al. [9]), i.e.,

$$
\left.t_{i}^{\mathrm{ff}}\right|_{\Gamma_{\mathrm{ff}}}=\left.\sigma_{i 2}^{\mathrm{ff}}\right|_{\Gamma_{\mathrm{ff}}}=0 \text {. }
$$

Finally, the displacements and stress fields that are scattered by the free-surface boundary and by the crack's line $S$ are denoted as $u_{i}^{\mathrm{sc}}(x, \omega)$ and $\sigma_{i j}^{\mathrm{sc}}(x, \omega)$, respectively.

\subsection{Boundary conditions}

The mechanical model of Gurtin [5], assumes that the interface layer $S$ between the crack and surrounded matrix has zero thickness and its own elastic properties. In addition, the surface stress along this interface layer is given by

$$
\sigma_{i j}^{S}=\tau_{0} \delta_{i j}+\partial E / \partial \varepsilon_{i i}^{S} \text {. }
$$

In the above, $E$ is the deformation-dependent surface energy density, $\varepsilon_{i i}^{S}$ is the strain tensor along surface $S$ and $\tau_{0}$ is the residual surface tension under unstrained conditions along $S$, which induces an additional static deformation. This tension is 
usually ignored in dynamic analysis, because its static nature does not influence the response of the solid under transient loads. It is assumed that the surface layer $S$ has zero thickness, but is otherwise elastic and isotropic with surface Lam'e constants $\lambda^{s}$ and $\mu^{S}$ whose numerical values are available in the literature. Additionally, it is assumed that the interface $S$ is a coherent, perfectly bonded layer, where the strain $\varepsilon_{l l}^{S}$ in the tangential direction is equal to the tangential strain in the bulk matrix, i.e., $\varepsilon_{l l}^{S}=\varepsilon_{l l}^{M}$. In this case, the condition $\varepsilon_{l l}^{S}=u_{n} / \bar{\rho}+\partial u_{l} / \partial l$ is a priori satisfied [5], where $\bar{\rho}$ is the curvature radius of the boundary $S$.

The following conditions for the stresses are satisfied along the tangential and normal directions of the interface $S$, i.e., the local coordinate system described by the normal $n$ and tangential $l$ vector components as

$$
\sigma_{n l}^{M}=-\frac{\partial \sigma_{l l}^{S}}{\partial l}, \text { and } \sigma_{n n}^{M}=\frac{\sigma_{l l}^{S}}{\bar{\rho}} \text { along } S .
$$

The boundary condition (7) can be rewritten with respect to the tractions developed along S, see Dong and Pan [3], Rangelov and Dineva [10] as follows:

$$
\left(\begin{array}{l}
t_{1}^{M} \\
t_{2}^{M}
\end{array}\right)=T^{S}\left(\begin{array}{l}
u_{1} \\
u_{2}
\end{array}\right) \text { along } S
$$

where

$$
\begin{gathered}
T^{S}=T_{1}+\frac{\partial}{\partial l} T_{2}+\frac{\partial^{2}}{\partial l^{2}} T_{3} ; T_{1}=\frac{1}{\bar{\rho}^{2}} N\left(\begin{array}{cc}
-\alpha^{S} & 0 \\
-\alpha^{S} \bar{\rho}_{, l} & 0
\end{array}\right) N^{\prime} ; T_{2}=N\left(\begin{array}{cc}
0 & -\beta^{S} \\
\beta^{S} & 0
\end{array}\right) N^{\prime} ; \\
T_{3}=N\left(\begin{array}{cc}
0 & 0 \\
0 & \alpha
\end{array}\right) N^{\prime} ; N=\left(\begin{array}{cc}
n_{1} & -n_{2} \\
n_{2} & n_{1}
\end{array}\right) .
\end{gathered}
$$

In the above, $\alpha^{S}=\lambda^{S}+2 \mu^{S}, \beta^{S}=\frac{1}{\bar{\rho}} \alpha^{S}, n_{i}=-n_{i}^{M}$, and $\frac{\partial}{\partial l}, \frac{\partial^{2}}{\partial l^{2}}$, respectively, are the first and second tangential derivatives, while the traction components that developed in the bulk matrix are denoted by $t_{k}^{M}=\sigma_{k j}^{M} n_{j}$, and $N^{\prime}$ is the transpose of matrix $N$. Note that if $\lambda^{S}=\mu^{S}=0$, the boundary condition (8) degenerates to the classical traction-free boundary condition for a line crack without surface elasticity effects.

\section{Boundary value problem and boundary integral equation}

The BVP is described by the governing Equation (3), the traction-free boundary condition along the free surface of the graded half-plane, Equation (5), by the boundary conditions along the nano-crack, Equation (8), and by the Sommerfeld radiation condition. The BVP problem is reformulated using a frequency-dependent, non-hypersingular traction-based Boundary Integral Equation (BIE) along the crack line $S$. Following $[2,10]$ we obtain the BIE, which is now an Integro-Differential 
Equation (IDE), for the unknown displacement $u_{j}$ on $S$ as follows:

$$
\gamma_{r j}\left(t_{r}^{\mathrm{ff}}(x, \omega)-t_{r}^{M}(x, \omega)\right)=C_{i j k l}(x) n_{i}(x) \int_{S}\left[\left(\Sigma_{\eta p k}^{*}(x, \zeta, \omega) u_{p, \eta}^{\mathrm{sc}}(\zeta, \omega)-\right.\right.
$$

$$
\left.\left.-\rho \tilde{\omega}^{2} U_{q k}^{*}(x, \zeta, \omega) u_{q}^{\mathrm{sc}}(\zeta, \omega)\right) \delta_{\lambda l}-\Sigma_{\lambda p k}^{*}(x, \zeta, \omega) u_{p, l}^{\mathrm{sc}}(\zeta, \omega)\right] n_{\lambda}(\zeta) d S_{\zeta}, \quad x \in S .
$$

In the above, $\gamma_{r j}$ is the jump term depending on the local geometry at the source point on the surface, namely $x \in S$. Function $U_{q k}^{*}(x, \zeta, \omega)$ is the Green's function for a point load acting in graded half-plane $x_{2} \leq 0$, see R a n g elo v and M a n ol is [13], while the corresponding stress tensor is $\Sigma_{i j q}^{*}(x, \zeta, \omega)=C_{i j k l}(x) \frac{\partial U_{q k}^{*}(x, \zeta, \omega)}{\partial \zeta_{l}}$ $\Sigma_{i j q}^{*}(x, \zeta, \omega)=C_{i j k l}(x) \frac{\partial U_{q k}^{*}(x, \zeta, \omega)}{\partial \zeta_{l}}$. Finally, the pair $(x, \zeta)$ represents the position vectors of the source and receiver points acting on the interior surfaces. Note that the use of the Green's function yields an integro-differential Equation (9) over the line of the nano-crack.

Once displacements along the surface $S$ are obtained, the displacements and stresses for the scattered wave field at any observation point $x \notin S$ in the bulk material, including the displacement along the free horizontal line $x_{2}=0$, can all be recovered using the corresponding integral representation formulae

$$
\begin{gathered}
u_{j}^{\mathrm{sc}}(x, \omega)=-\int_{S} \Sigma_{k i j}^{*}(x, \zeta, \omega) u_{k}^{\mathrm{sc}}(\zeta, \omega) n_{i} d S_{\zeta}, x \in\left\{x_{2} \leq 0\right\} \backslash S, \\
\sigma_{p q}^{\mathrm{sc}}(x, \omega)=-C_{p q k l}(x) \int_{S} \Sigma_{i j k, l}^{*}(x, \zeta, \omega) u_{i}^{\mathrm{sc}}(\zeta, \omega) n_{j} d S_{\zeta}, x \in\left\{x_{2} \leq 0\right\} \backslash S .
\end{gathered}
$$

The Stress Concentration Factors (SCF) close to the blunt crack-tip are evaluated as follows:

$$
\begin{aligned}
& F_{I}^{ \pm}\left(x_{1},-d, \omega\right)=\frac{\sigma_{22}\left(x_{1},-d, \omega\right)}{\sigma_{22}^{\mathrm{ff}}\left(x_{1},-d, \omega\right)} \sqrt{2 \pi\left(x_{1} \mp a\right)}, \\
& F_{I I}^{ \pm}\left(x_{1},-d, \omega\right)=\frac{\sigma_{12}\left(x_{1},-d, \omega\right)}{\sigma_{12}^{\mathrm{ff}}\left(x_{1},-d, \omega\right)} \sqrt{2 \pi\left(x_{1} \mp a\right)} .
\end{aligned}
$$

Note that the normalized SCFs are defined as $F_{I}^{ \pm}=\left|F_{I}^{ \pm}\right|$and $F_{I I}^{ \pm}=\left|F_{I I}^{ \pm}\right|$. For the normal incident $\mathrm{P}$-wave, both left and right SCFs are equal and we use notation $F_{I}^{*}=F_{I}^{ \pm}, F_{I I}^{*}=F_{I I}^{ \pm}$.

\section{Numerical solution}

The numerical solution of the problem stated above is described in what follows, with all the necessary programming for recovering numerical results performed on the symbolic mathematics package Mathematica [6] 


\subsection{Numerical scheme}

The numerical technique developed for solving the IDE of Equation (9) for the nanocracked, viscoelastic, graded bulk material is based on the free surface, half-plane Green's function, see Rangelov, Dineva and Manolis [11]. The following procedural steps are now defined:

(a) Discretization of the crack surface and approximation of the nodal unknowns. No discretization is required along the free surface of the half-plane because the analytically derived Green's function satisfies the traction-free condition. The mesh employed consists of 14 quadratic-type Boundary Elements (BE) along the blunt nano-crack S. Special Quarter-Point Boundary Elements (QP-BE) are used for correctly modelling the crack-tip zones, a process well-defined in linear fracture mechanics.

(b) Numerical evaluation of the singularities in the kernels of the surface integrals. The following type of singularities exists in this numerical scheme: (i) weak singularity of $\ln r$ type, and (ii) strong singularity of $1 / r$ type, where the corresponding integrals are understood in the Cauchy principal value sense.

(c) Gaussian quadrature schemes for evaluating of all regular and singular integrals, including verification of the accuracy of computation. Also, a Quasi Monte Carlo scheme is used for evaluating the integrals with infinite boundaries that appear in the Green's function. This point demands the use of more powerful computers together with development of more scalable algorithms in the computational scheme based on the boundary integral equations.

(d) Assembly of the system of equations and formation of the BIEM influence matrices using the nodal collocation technique.

(e) Solution of the resulting algebraic systems of equations;

(f) Post-processing of the results, primarily by back-substitution of the boundary solutions in Equation (10) to obtain results in the interior of the half-plane.

\subsection{Numerical examples}

In the numerical examples that follow, we define a dimensionless frequency as $m=\omega d / C_{\mathrm{R}}$, where the Rayleigh wave speed is denoted as $C_{\mathrm{R}}=C_{S}[(0.826+1.14 v) /(1+v)]$, and $v=0.25$ is Poisson's ratio. Values for the material properties for the reference homogeneous bulk material are $\lambda_{0}=\mu_{0}=2.216 \times 10^{10} \mathrm{~Pa}$ for the Lamé constants and $\rho_{0}=2 \times 10^{3} \mathrm{~kg} / \mathrm{m}^{3}$ for the mass density. For the nano-crack, we have that $|S|=2.0428 \times 10^{-8} \mathrm{~m}$, and the end semi-circles $S^{l}, S^{r}$ have a radius $c=0.0375 a$, with $a=5 \times 10^{-9} \mathrm{~m}$. A dimensionless surface elasticity parameter $s=\alpha^{S} /\left(2 \mu_{0} c c_{S}\right)$, with $\alpha^{S}= \pm 6.091 \mathrm{~N} / \mathrm{m}$ is also defined. Values for $\alpha^{S}$ are usually determined by either atomic simulation techniques or by experiments. In our case, the value of $\alpha^{S}$ is taken from Sharma et al. [15]. The case $s=0$ corresponds to the blunt crack without surface effects. More specifically, parameter $c_{S}$ is the curvature radius in the QP-BEs used for correctly 
modelling the crack-tip zones. For the case where the blunt nano-crack approaches the line crack geometry, the radius of curvature $c_{S}$ in the QP-BEs increases proportionally; conversely, when the value of surface parameter decreases to 0 , we recover the case of the classical line crack. The normalized inhomogeneity parameter that defines the departure from an equivalent homogeneous medium is $b=2 a a_{2}$. The accuracy and convergence characteristics of the proposed numerical scheme is checked by keeping the well-known discretization rule of $\lambda_{\mathrm{SV}}>10 l_{\mathrm{BE}}$, where $\lambda_{\mathrm{SV}}$ is the SV-wave length and $l_{\mathrm{BE}}$ is the maximum size of the Boundary Elements (BE) used. Detailed verification studies for a nano-crack in a graded half-plane and in an viscoelastic of Zener type material under time-harmonic plane waves are presented in the authors' publications of $\mathrm{R}$ a $\mathrm{ng}$ e lo v, D in e v a and M a n ol is $[11,12]$.

In what follows, we present a parametric study illustrating the sensitivity of both the local SCF near the nano-crack and of the diffracted and scattered wave fields along the free surface of the graded half-plane to key model parameters. At first, Fig. 2 depicts the normalized SCF-I at a nano-crack in a homogeneous viscoelastic full plane (Fig. 2a, c, e) and in a homogeneous viscoelastic half-plane (Fig. 2b, d, f). The nano-crack is embedded at depth $d / 2 a=0.5$ and all plots are in terms of the normalized frequency $m$ of a normally incident P-wave. The following model parameters are kept fixed: The values for the Zener model are $\alpha=0.5$ and $p=q=1$ (Fig. 2a, b), or $p=5, q=1$ (Fig. 2c, d), or $p=1, q=5$ (Fig. 2e, f). Next, the surface parameter values are $s=0 ; 0.04 ; 1$. Fig. 2a-f illustrate clearly the magnitude of wave scattering by the free-surface of the viscoelastic homogeneous half-plane on the zones of the dynamic local SCF near the nano-crack. This scattering phenomenon by the free surface of the half-plane has a strong influence on both amplitudes and resonance frequencies in the frequency interval under consideration. Fig. 2a-f all clearly show that dynamic SCF depend on the material viscosity parameters $p$ and $q$, on the frequency of the incident wave, on the surface elasticity effects due to existence of a nano-crack and on the scattering effects due to presence of a freesurface boundary.

The effect of viscoelasticity can be seen in Fig. 3, where the normalized SCF-I for a nano-crack embedded at $d / 2 a=1.5$ in a quadratically graded half-plane, which is either elastic isotropic (Fig. 3a) or viscoelastic isotropic with Zener model parameters $p=5, q=1$ (Fig. 3b), versus normalized frequency $\mathrm{m}$ of a normally incident P-wave. In Fig. 3, the following fixed parameters values are used: The dimensionless surface parameter is $s=0.14$ and the normalized material inhomogeneity parameter values are $b=-0.5$ and $b=-1$. Fig. 3 clearly shows that both material inhomogeneity and viscosity, interacting with the frequency of the dynamic load, are able to change the amplitude-frequency characteristics of the SCF near nano-crack in measurable way. Essentially, these two characteristics work in opposite ways, with inhomogeneity resulting in continuous scattering of the incoming wave resulting in localized peaks, while viscoelasticity smoothens the wave motion. 

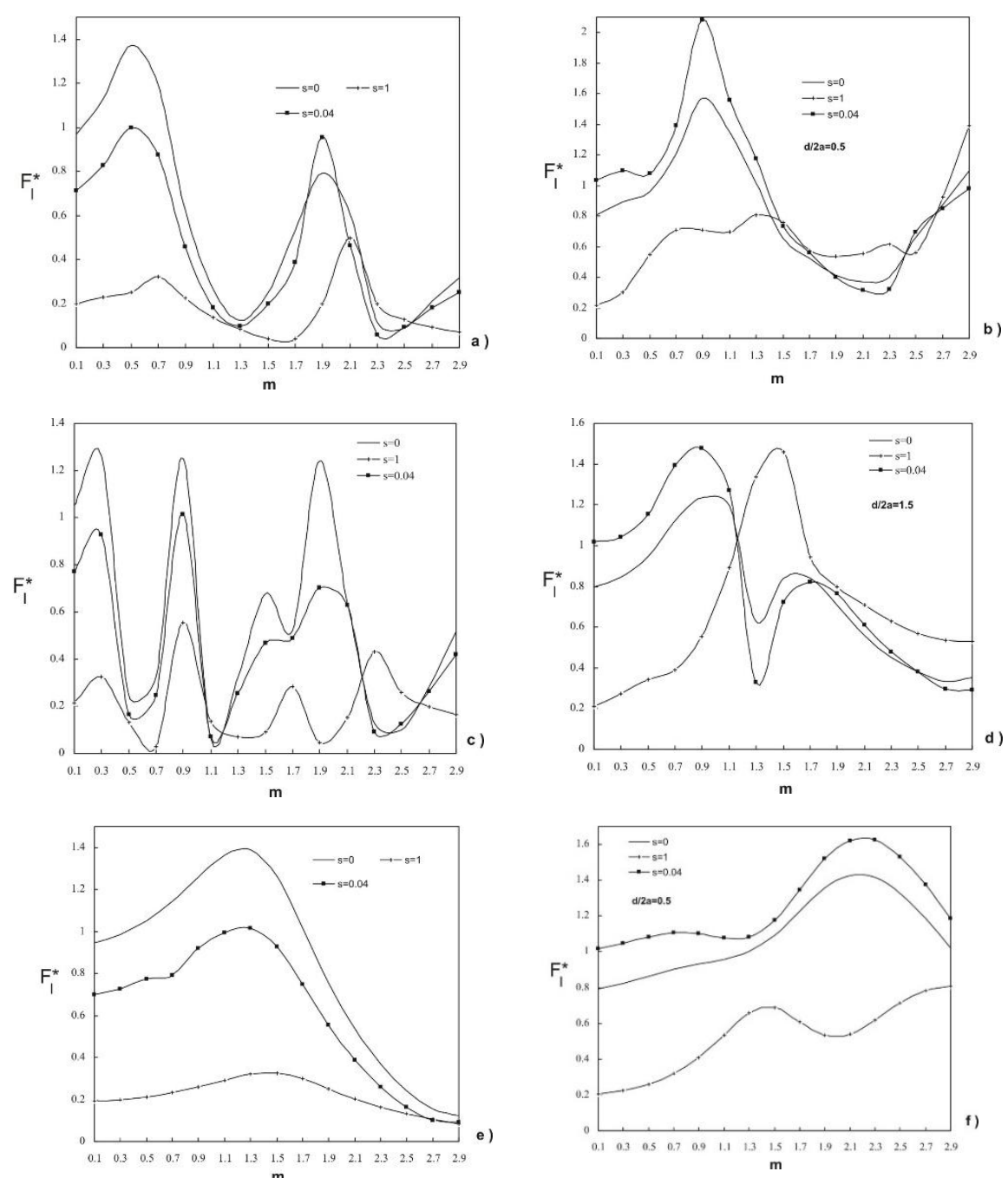

Fig. 2. Normalized SCF-I of a nano-crack in (a), (c), (e) a viscoelastic full plane and (b), (d), (f) in a viscoelastic half-plane for a crack depth $d / 2 a=0.5$ versus normalized frequency $\boldsymbol{m}$ of a normally incident P-wave. Zener model fixed values are $\alpha=0.5$ and $p=q=1$ in (a), (b), or $p=5, q=1$ in

(c), (d), or $p=1, q=5$ in (e), (f). Fixed values of the surface parameters are $s=0,0.04,1$
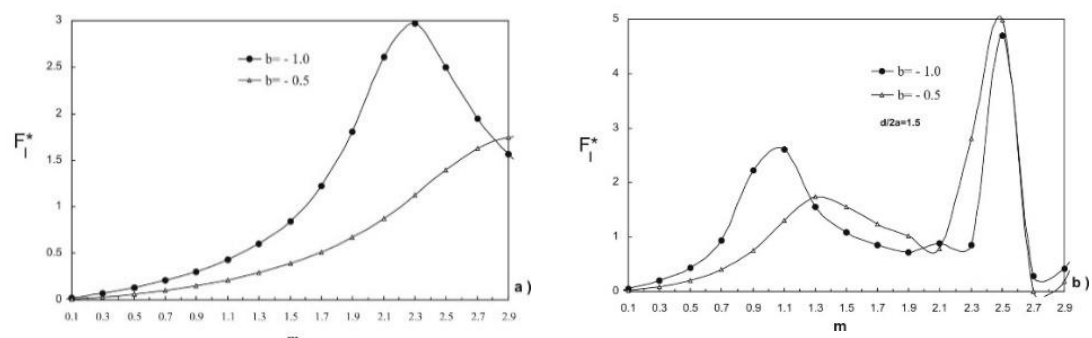

Fig. 3. Normalized SCF-I for a nano-crack embedded at depth $d / 2 a=1.5$ in an inhomogeneous elastic (a), and viscoelastic (b) with parameter values of $p=5, q=1$ half-plane for fixed values of the surface parameter $s=0.14$ and the inhomogeneity parameter $b=-1.0,-0.5$ 
Finally, Fig. 4 presents the normalized, horizontal displacement amplitude $\left|u_{l}\right|$ of the scattered surfaces wave at observer point $\mathrm{A}(-a, 0)$ versus normalized frequency $m$ of a normally incident $P$-wave in the homogeneous elastic (Fig. 4a) and in the homogeneous viscoelastic (Fig. 4b) half-plane with an embedded nano-crack at depth $d / 2 a=1.5$. As before, the following surface parameter values are used: $s=0 ; \pm 0.029$. For the viscoelastic case, the Zener material parameters are $\alpha=0.5$, $p=1, q=5$. Again, the sensitivity of the scattered and diffracted wave fields that develop in the bulk material to viscosity, to surface elasticity effects because of the nano-crack and to the frequency of the incoming dynamic excitation are clearly illustrated in Fig 4. All things being equal, we see that the localized displacement response peaks in the inhomogeneous material occur at normalized frequencies that are quite higher than those that are observed when the material is viscoelastic.
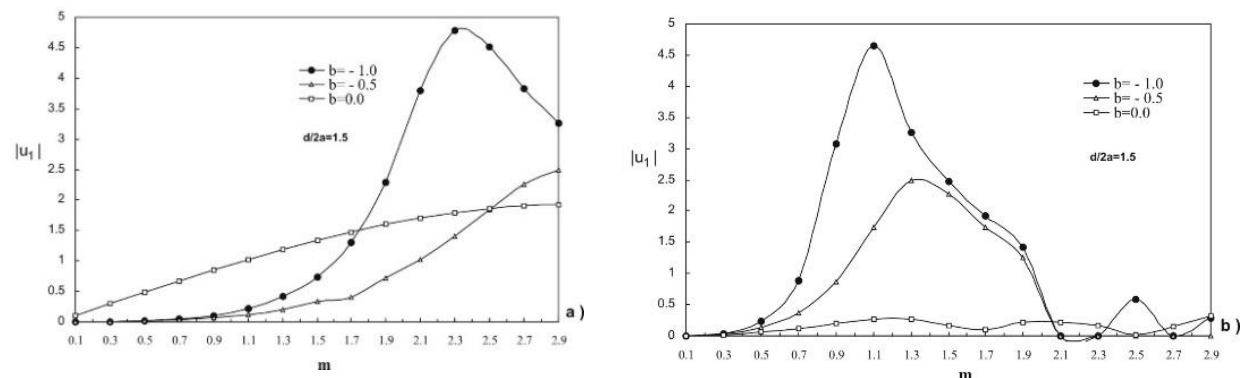

Fig. 4. Normalized displacement amplitude $\left|u_{1}\right|$ at observer point $A(-a, 0)$ versus normalized frequency $m$ in a graded elastic (a), and viscoelastic (b), with $p=5, q=1$ half-plane containing a nano-crack at depth $d / 2 a=1.5$. Fixed values for the surface parameters are $s=0.14$ and for the inhomogeneity parameter are $b=-1.0,-0.5$

\section{Conclusions}

A two-dimensional, nano-cracked solid with free-surface embedded in a quadratically graded, viscoelastic material is studied under time-harmonic conditions. The non-hypersingular traction BIEM formulation is used in conjunction with both the closed form, frequency dependent Green's function for a graded halfplane and the surface-elasticity model of Gurtin-Murdoch. Following the mathematical formulation and numerical solution, an extensive parametric study was conducted to investigate the sensitivity of the resulting scattered elastic wave and stress concentration fields to the following key problem parameters: Material inhomogeneity, viscosity, and interaction effects between the crack and the free surface of the material, all under the presence of incident waves.

Acknowledgements: The authors are grateful for support provided under research Grant No BG05M2OP001-1.001-0003, financed by the Bulgarian Science and Education for Smart Growth Operational Program (2014-2020) and co-financed by the European Union through the European Structural and Investment Funds and by the bilateral Bulgarian-Greek Scientific Exchange project between the Bulgarian Academy of Sciences and Aristotle University. 


\section{References}

1. Arro y o, M. T. V., T. B e ly t s ch kh o. An Atomistic-Based Finite Deformation Membrane for Single Layer Crystalline Films. - J. Mech. Phys. Solids, Vol. 50, 2002, pp. 1941-1977.

2. Dineva, P. S., T. R angelov. Wave Scattering by Cracks at Macro- and Nano-Scale in Anisotropic Plane by BIEM. - J. Theor. Appl. Mech., Vol. 46, 2016, No 4, pp. 19-35.

3. D o n g, C. Y., E. P a n. Boundary Element Analysis of Inhomogeneities of Arbitrary Shapes with Surface and Interface Effects. - Eng. Anal. Bound. Elem., Vol. 35, 2011, pp. 996-1002.

4. Gradshteyn, I. S., I. M. Ryzhik. Tables of Integrals, Series, and Products. New York, Academic Press, 1965.

5. Gurti n, M. E., A. I. Murdoch. A Continuum Theory of Elastic Material Surfaces. - Arch. Ration. Mech. Anal., Vol. 57, 1975, pp. 291-323.

6. MATHEMATIKA. Champaign, Illinois, Wolfram Research, 1987.

7. Ma in ardi, F. Fractional Calculus and Waves in Linear Viscoelasticity: An Introduction to Mathematical Models. London, UK, Imperial College Press, 2010.

8. Makrou, A. A., G. D. Manolis. A Fractional Derivative Zener Model for the Numerical Simulation of Base Isolated Structures. - Bull. Earthquake Eng., Vol. 14, 2016, No 1, pp. 283-295.

9. Man olis, G. D., P. S. Di n e va, T. V. R a n ge lov, F. W u t t ke. Seismic Wave Propagation in Non-Homogeneous Elastic Media by Boundary Elements. - In: Solid Mechanics and Its Applications. Vol. 240. Cham, Switzerland, Springer International Publishers, 2017.

10. R a n g e $1 \mathrm{o} \mathrm{v}, \mathrm{T}$. V., P. S. D i n e v a. Dynamic Fracture Behavior of a Nano-Crack in a Piezoelectric Plane. - ZAMM, Z. Angew. Math. Mech., Vol. 97, 2017, No 11, pp. 1393-1405.

11. R a n g e l o v, T. V., P. S. D i n e v a, G. D. M a n o l i s. BIEM Analysis of a Graded Nano-Cracked Elastic Half-Plane under Time-Harmonic Waves. - ZAMM, Vol. 100, 2020, 100:e202000021. https://doi.org/10.1002/zamm.202000021

12. R a ngelov, T. V., P. S. Dineva, G. D. Manolis. Dynamic Response of a Cracked Viscoelastic Anisotropic Plane Using Boundary Elements and Fractional Derivatives. J. Theor. Appl. Mech., Vol. 48, 2018, No 2, pp. 24-49.

13. R a n ge lov, T. V., G. D. M a n o li s. Time-Harmonic Elastodynamic Green's Function for the Half-Plane Modelled by a Restricted Inhomogeneity of Quadratic Type. - J. Mech. Mater. Strut., Vol. 5, 2010, No 6, pp. 909-924.

14. S c h a n z, M. Wave Propagation in Viscoelastic and Poroelastic Continua: A Boundary Element Approach. Lecture Notes in Applied Mechanics. Vol. 2. Berlin, Springer, 2001.

15. S h a r m a, P., S. G a n t i, N. B h a t e. Effect of Surfaces on the Size-Dependent Elastic State of Nano-Inhomogeneities. - Appl. Phys. Lett., Vol. 82, 2003, pp. 535-537.

Received: 15.09.2020; Second Version: 23.10.2020; Accepted: 30.10 .2020 